\title{
Propuesta de diseño de un sistema de energía solar fotovoltaica. Caso de aplicación en la ciudad de Bogotá
}

\author{
Design proposal for a photovoltaic solar energy system. Case of application in the \\ city of Bogotá
}
Proposta de design para um sistema de energia solar fotovoltaica. Caso de aplicação na cidade de Bogotá

Sebastián Salamanca-Ávila ${ }^{1}$

Recibido: mayo de 2017

Aceptado: agosto de 2017

Para citar este artículo: Salamanca-Ávila, S. (2017). Propuesta de diseño de un sistema de energía solar fotovoltaica. Caso de aplicación en la ciudad de Bogotá. Revista Científica, 30 (3), 263-277. Doi: https://doi. org/10.14483/23448350.12213

\section{Resumen}

La energía solar es un recurso renovable, es decir, está siempre disponible, no se agota y se puede aprovechar en cualquier momento gracias a que es posible almacenarla. Las difíciles condiciones medioambientales, la contaminación y, de otro lado, el avance tecnológico en el desarrollo de celdas solares cada vez más eficientes, han contribuido a que en la actualidad se promueva el uso de las energías renovables como la energía solar. Adicionalmente, se ha incrementado el uso de estos sistemas pues el nivel de contaminación que produce es muy bajo y los costos de instalación se recuperan al reducir la facturación del consumo de energía prestado por empresas de servicios públicos. Por lo tanto, el uso de sistemas fotovoltaicos es un tema de mucho interés en la actualidad e implementar este tipo de sistemas aislados en la ciudad de Bogotá es posible.

Palabras clave: Energía solar; panel solar fotovoltaico; renovables; MPPT.

\begin{abstract}
Solar energy is a renewable resource, that is, it is always available, it is not exhausted and you can take advantage of that energy from the sun at any time thanks to its storage. The difficult environmental conditions, pollution and, on the other hand, the technological advance in the development of increasingly efficient solar cells, has contributed to the current promotion of the use of renewable energy such as solar energy. In addition, the use of these systems has increased since the level of pollution is very low and installation costs are recovered by reducing the billing of energy consumption, provided by utilities. Therefore, the use of photovoltaic systems is a topic of great interest today, and to implement this type of isolated photovoltaic systems in the city of Bogota is possible.
\end{abstract}

Keywords: Solar energy; photovoltaic solar panel; renewable; MPPT. 


\section{Resumo}

A energia solar é um recurso renovável, ou seja, está sempre disponível, não se esgota, podendo ser aproveitada em qualquer momento porque é possível armazená-la. As difíceis condições ambientais, a contaminação e, por outro lado, o progresso tecnológico no desenvolvimento de células solares cada vez mais eficientes, tem contribuído a que na atualidade se promova o uso das energias renováveis como a energia solar. Adicionalmente, tem se aumentado o uso destes sistemas devido a que o nível de contaminação é muito baixo e os custos de instalação se recuperam a través da redução do consumo de energia, fornecida por empresas de serviços públicos. Portanto, o uso de sistemas fotovoltaicos é um assunto de muito interesse na atualidade, e implementar este tipo de sistemas fotovoltaicos isolados na cidade de Bogotá é possível.

Palavras-chaves: Energía solar, panel solar fotovoltaico, renovables, MPPT.

\section{Introducción}

El acceso a la energía eléctrica es un factor importante en el desarrollo de la humanidad (Valeret al., 2016). Hoy en día, la producción de energía eléctrica depende en gran medida de los recursos fósiles disponibles y la posibilidad de acceso a la red eléctrica (Rosso-Cerón y Kafarov, 2015). Durante varias décadas el desarrollo de las poblaciones ha dependido estrictamente de la capacidad de uso de combustibles fósiles para la obtención de energía, lo que ha provocado un gran impacto negativo al medio ambiente con respecto a las emisiones de dióxido de carbono $\left(\mathrm{CO}_{2}\right)$ en la atmosfera (Ben y Ben, 2017; Ghasemi Mobtaker et al., 2016). De este problema ambiental ha surgido el concepto Green Economy, el cual ha ganado mucha importancia entre los académicos y los encargados de hacer políticas en lo que respecta a temas de producción de energía (Gasparatos et al., 2017; Cao et al., 2017).

El uso y producción de energía renovable no supera el $20 \%$ en el actual consumo a nivel mundial (Gasparatos et al., 2017). Mientras tanto, la demanda de energía incrementa excesivamente a causa del aumento de la población y el desarrollo industrial, por este motivo se promueven políticas económicas, ambientales y sociales orientadas a la búsqueda de nuevas formas de suplir las necesidades energéticas de la población (Kannan y Vakeesan, 2016; Peralta y Eduardo, 2011). Es por esto que ya existen varios planes liderados por algunos países, como los miembros de la UE, los cuales tienen el propósito para el 2020 de suplir con fuentes de energía renovables más del $20 \%$ de la demanda de energía eléctrica (Gasparatos et al., 2017; Wahyuni et al.,2015).

Algunas de las energías renovables que menor impacto provocan al medio ambiente son: la eólica, la hidroeléctrica, la bioenergía (Kannan y Vakeesan, 2016; Gasparatos et al., 2017) y la energía solar, en la que se hará énfasis para explicar sus beneficios y aplicaciones.

\section{Energía solar}

La energía solar puede ser la mejor opción para el futuro de la humanidad porque es el recurso más abundante de energía renovable. El sol emite cerca de $3.8 \times 10^{23} \mathrm{~kW}$, de los cuales aproximadamente $1.8 \times 10^{14} \mathrm{~kW}$ son interceptados por nuestro planeta (Kannan y Vakeesan, 2016); la energía solar llega en forma de luz y calor. La mayor parte de esta se pierde debido a la dispersión, la reflexión y la absorción por las nubes. Estudios han demostrado que la demanda global de energía puede ser proporcionada por el sol (Lewis, 2007; Lúcio et al., 2016). Otra razón por la cual esta es la energía del futuro es que su utilización no tiene algún impacto perjudicial para el medio ambiente y no afecta el equilibrio de los ecosistemas, comparado con la explotación de los recursos fósiles que claramente causan mucho daño (Kannan y Vakeesan, 2016; Ghasemi Mobtaker et al., 2016; Lúcio et al., 2016).

La energía solar aprovecha el poder del sol para generar electricidad, ya sea directamente a través de células fotovoltaicas (PV) o a través de medios de energía solar concentrada (CSP). Las tecnologías 
CSP utilizan matrices de espejos que rastrean el sol y reflejan continuamente sus rayos hasta el punto heliostatos para calentar un líquido de trabajo, que luego se utiliza para generar electricidad en una turbina convencional (Zhou et al., 2017; Senturk y Eke, 2017; Chandel, Nagaraju Naik, y Chandel, 2015). Otras nuevas tecnologías de energía solar utilizan también la luz solar concentrada en PV de mayor calidad y eficiencia. Generalmente, se requiere de grandes áreas para ser eficaces, mientras que los paneles solares fotovoltaicos pueden ser distribuidos y montados en cualquier superficie expuesta al sol, lo que los hace ideales para la integración en el medio ambiente urbano o cualquier otro terreno (Gasparatos et al., 2017).

Los sistemas fotovoltaicos (PV) convierten la luz solar directamente en energía eléctrica sin la interposición de cualquier motor térmico. La base de la tecnología fotovoltaica se ha establecido sobre el principio de efecto fotovoltaico que fue observado por primera vez en 1839 por Edmons Becquerel. Sin embargo, fue necesario otro medio siglo para construir las células fotovoltaicas para su uso práctico. La primera práctica con celdas solares fue realizada en 1954, en Bell Telephone Laboratories por Calvin Fuller y Gerald Pearson et al., 2016). Posteriormente, se realizaron más investigaciones y desarrollo de obras de tecnología fotovoltaica para suministrar energía a los satélites espaciales; además, tras la crisis energética de la década de 1970 se incrementó el interés público por la aplicación de la energía solar fotovoltaica como fuente de energía eléctrica alternativa (Olaya, Arango-Aramburo y Larsen, 2016; Islam et al., 2016).

\section{Sistemas fotovoltaicos}

La energía solar fotovoltaica es una fuente de energía que produce electricidad de origen renovable obtenida directamente a partir de la radiación solar mediante un dispositivo semiconductor denominado célula fotovoltaica, o bien mediante una deposición de metales sobre un sustrato denominada célula solar de película fina (Aguilar, Aledo y Quiles, 2016).

Las células fotovoltaicas que se fabrican con materiales semiconductores son el componente básico de un sistema fotovoltaico. Un número de células solares conectadas eléctricamente entre sí forman un módulo fotovoltaico. Múltiples módulos pueden ser conectados para formar un arreglo, a su vez, estos arreglos pueden ser conectados entre sí en paralelo o serie para obtener más corriente o más potencia (Islam et al., 2016).

En las últimas décadas, los sistemas fotovoltaicos se han convertido en una forma de producción de energía eléctrica efectiva, especialmente en lugares con buena radiación (Yahyaoui, Chaabene, y Tadeo, 2015), gracias a que su instalación es fácil y el mantenimiento posterior es de bajo costo, lo que permite que los sistemas fotovoltaicos se usen con más frecuencia (Abella, 2016).

\section{Componentes de sistemas PV}

\section{Bloque de generación}

El bloque de generación está conformado por los paneles fotovoltaicos, donde su número y tipo de conexión existente entre ellos depende de varios factores como: el valor promedio de la insolación del lugar, la carga y la máxima potencia nominal de salida del panel (García, 2016).

\section{Bloque de acumulación}

El bloque de acumulación es la parte del sistema fotovoltaico encargado de almacenar y controlar la carga y descarga del sistema (García, 2016). Está conformado por los siguientes componentes:

\section{Banco de baterías.}

Por lo general son baterías de ciclo profundo, las cuales están diseñadas para soportar niveles de descarga profundos durante muchos ciclos de carga y descarga. 


\section{Banco de baterías.}

Evita la descarga de las baterías a través de los paneles durante le noche, cuando el voltaje de salida del panel PV es nulo.

\section{Fusibles o llaves de protección.}

Protegen las baterías y son incorporadas al sistema como un elemento de seguridad.

4. Medidor de carga.

Dispositivo que permite conocer el estado de carga del banco.

\section{Bloque de carga}

El bloque de carga está encargado de suministrar la energía producida por los paneles solares a los equipos que requieran energía eléctrica (García, 2016) y lo conforman:

\section{Inversor}

Su función es convertir la corriente continua proveniente de las baterías o directamente del panel en corriente alterna para su aprovechamiento.

\section{Cableado}

Es lo más básico del sistema y su selección tiene un rol importante en la reducción de pérdidas de energía.

\section{Diseño de sistemas PV}

El diseño óptimo de los sistemas PV es un factor muy importante en todas las instalaciones de estos. Este diseño óptimo muchas veces depende de la variable de radiación solar (Zhou et al., 2017; Muhsen, Ghazali y Khatib, 2016). Varias metodologías han sido reportadas en la literatura para el dimensionamiento de los sistemas fotovoltaicos, algunas de estas son: el diseño intuitivo, analítico y el diseño basado en métodos numéricos. El diseño por método numérico es el más efectivo y recomendado ya que parte de un diseño intuitivo el cual, por medio de cálculos, determina la configuración óptima (García,2016; Dhiaa Halboot Muhsen et al., 2016; Muhsen, Ghazali, Khatib, Abed, y Natsheh, 2016). Otro punto importante en la selección del sistema fotovoltaico es el factor económico, ya que es muy importante seleccionar la configuración más económica que satisfaga la necesidad de energía que se va a suplir con el sistema PV (Fouda, Nada y Elattar, 2016; Valer et al., 2016). El gran desarrollo computacional y la tecnología han permitido realizar avances importantes en el diseño de sistemas fotovoltaicos con la ayuda del sistema de métodos numéricos, ya que itera muchas opciones las cuales son posibles soluciones al problema, y permite escoger la más óptima (García, 2016; Cao et al., 2017).

\section{Diseño para un sistema aislado}

La metodología presentada a continuación es del tipo de metodología de diseño de sistemas fotovoltaicos aislados que se aplica en España. Esta fue mostrada a los estudiantes del curso de energías renovables de la Universidad Politécnica de Valencia, en España, por parte del ingeniero Eugeni García (García, 2016).

\section{Determinación del perfil de carga:}

El perfil de carga nos da información sobre la simultaneidad de los consumos y sirve para calcular los sistemas de acondicionamiento de potencia y de distribución. Para hacer el perfil del consumo hay que tener en cuenta los hábitos del lugar estudiando para cada caso en concreto.

\section{Estimación del consumo:}

Es el consumo diario de energía eléctrica, el cual debe ser suministrado por el sistema fotovoltaico, y se tiene en cuenta el consumo energético de CC y AC. 


$$
E_{C C}=\sum \# \text { Eqip } * \text { Horas } * P_{E q} \quad(\text { Ecuación } 1)
$$

La ecuación 1 se refiere a la estimación de consumo para equipos de CC donde \#Eqip es el número de equipos de las mismas características, Horas es la cantidad de horas que se prevé que esté en funcionamiento el equipo y, por último, $P_{E q}$ es la potencia nominal de cada equipo de CC conectado.

$$
E_{A C}=\sum \# \text { Eqip } * \text { Horas } * P_{E q} \quad(\text { Ecuación 2) }
$$

En la ecuación 2 las variables son exactamente iguales que en la 1 , solo que se aplican para los equipos de AC.

La energía total consumida por la carga es la suma de la energía consumida por los dispositivos de CC y la consumida por los dispositivos de AC.

$$
E_{\text {Cargatotal }}=E_{C C}+E_{A C} \quad \text { (Ecuación 3) }
$$

\section{Estimación de pérdidas:}

La energía que se genera por los paneles debe tomar en consideración las pérdidas de energía anticipadas en el sistema (cableado, control de carga, inversor y baterías).

$$
\eta_{T}=\eta_{B} * \eta_{i n v} * \eta_{R} * \eta_{X}
$$

(Ecuación 4)

- $\eta_{B}$ : eficiencia debido al rendimiento de la batería que típicamente puede oscilar entre $75 \%$ y un $90 \%$.

- $\eta_{\text {inv }}$ : eficiencia debido al rendimiento del inversor utilizado (si lo hay), es decir, principalmente en instalaciones de $220 \mathrm{~V}$. Los valores por defecto suelen oscilar entre el $85 \%$ y el $98 \%$.

- $\quad \eta_{R}$ : eficiencia debido al rendimiento del regulador empleado. Suele depender de la tecnología utilizada, pero, si no se conoce, se escoge un valor por defecto del $90 \%$.

- $\eta_{X}$ : eficiencia que considera las pérdidas no contempladas:
- Temperatura.

- Pérdidas por dispersión de parámetros y suciedad.

- Las pérdidas por errores en el seguimiento del punto de máxima potencia.

- Cableado.

\section{Dimensionamiento de los paneles fotovoltaicos:}

Este dimensionamiento implica calcular la energía total necesaria a generar (considerando la estimación de pérdidas) y con base en la insolación del lugar, determinar la cantidad de paneles y la forma de conexión (serie y paralelo).

El coeficiente $\gamma$ es un factor de seguridad para afrontar la degradación de potencia y prestaciones de los diferentes componentes del sistema fotovoltaico.

$$
E_{g e n}=\gamma\left(\frac{E_{A C}}{\eta_{T A C}}+\frac{E_{C C}}{\eta_{T C C}}\right)
$$

(Ecuación 5)

Donde $E_{g e n}$ es la energía que se va a generar con el bloque generador, $\gamma$ es el factor de seguridad que suele ser 1.1, $\eta_{T A C}$ y $\eta_{T C C}$ son las eficiencias de cada uno de los sistemas de AC y CC y, por último, $E_{A C}$ y $E_{C C}$ son los consumos energéticos diarios de AC y CC.

\section{Dimensionamiento de los paneles fotovoltaicos:}

Si no se utiliza un regulador con seguimiento de punto de máxima potencia MPPT, el cual tiene como función determinar el punto de máxima eficiencia energética al instante en cualquier situación, deberá tenerse en cuenta que será entonces la batería la que marque la tensión del sistema.

$$
E_{\text {panel }}=W_{p(T)} * H S P * \frac{V_{n p}}{V_{p}}
$$

(Ecuación 6)

Donde $E_{\text {panel }}$ es la energía diaria generada por el panel, $W_{p(T)}$ es la potencia nominal o pico del panel corregida por temperatura, $H S P$ es la hora 
solar pico y, por último, $V_{n p}$ es la tensión nominal del panel y $V_{p}$ es la tensión pico del panel.

$$
W_{p(T)}=W_{p} *\left(1-\Delta T * \frac{c_{d}}{100}\right) \quad(\text { Ecuación 7) }
$$

Donde $\mathrm{T}$ es la temperatura de trabajo del panel en ${ }^{\circ} \mathrm{C}, \Delta T=\mathrm{T}-25^{\circ} \mathrm{C}$, que es el incremento por sobre los $25^{\circ} \mathrm{C}$ y, por último, $C_{d}$ es el valor porcentual del coeficiente de degradación.

\section{Determinación de la tensión nominal de la instalación:}

La tensión de funcionamiento se puede determinar a partir de la potencia de la instalación, que lógicamente está relacionada con la energía consumida. Se suelen emplear las tensiones estándar: $12 \mathrm{~V}$, $24 \mathrm{~V}, 48 \mathrm{~V}$ o $120 \mathrm{~V}$.

En general se recomienda:

- $12 \mathrm{~V}$ para potencias menores de $1.5 \mathrm{~kW}$.

- $24 \mathrm{~V}$ o $48 \mathrm{~V}$ para potencias entre $1.5 \mathrm{~kW}$ y 5 kW.

- $48 \mathrm{~V}$ o $120 \mathrm{~V}$ para potencias mayores de $5 \mathrm{~kW}$.

\section{Dimensionamiento de los paneles fotovoltaicos:}

\section{Cantidad total de paneles}

La cantidad total de paneles será la cantidad de paneles necesarios para poder abastecer a la carga.

$$
N_{T P}=\frac{E_{\text {gen }}}{E_{\text {panel }}}
$$

(Ecuación 8)

Donde $N_{T P}$ es el número total de paneles del bloque generador, $E_{g e n}$ es la energía diaria generada por el bloque y $E_{\text {panel }}$ es la energía diaria generada por el panel.

\section{Cantidad de paneles en serie}

La asociación de paneles en serie se hace con el fin de aumentar la tensión del bloque generador.

$$
N_{P S}=\frac{V_{n o m}}{V_{n p}}
$$

(Ecuación 9)

Donde $V_{\text {nom }}$ es la tensión nominal del sistema y $V_{n p}$ es la tensión nominal del panel.

\section{Cantidad de paneles en paralelo}

La asociación de paneles en paralelo se realiza con el fin de aumentar la capacidad de corriente del bloque generador una vez que la tensión nominal se alcanzó mediante la asociación de paneles en serie.

$$
N_{P P}=\frac{N_{T P}}{N_{P S}}
$$

(Ecuación 10)

Donde $N_{T P}$ es el número total de paneles del bloque generador y $N_{P S}$ es la cantidad de paneles en serie.

\section{Dimensionamiento del banco de baterías:}

El banco de baterías deberá suministrar la energía requerida por la carga cuando no haya sol, o en días nublados. Este debe acumular la energía necesaria para alimentar la carga durante los días sin sol y durante la noche. Además, esta energía debe contemplar las pérdidas producidas por los diversos componentes.

Para el cálculo de la capacidad del banco de baterías se necesita definir principalmente los siguientes parámetros:

- Daut: días de autonomía con baja o nula insolación.

- PDmax: profundidad máxima de descarga de la batería, que vendría dada por el fabricante de las baterías; por defecto se escoge un valor del $60 \%$ o $80 \%$.

- $\quad$ ¡D: eficiencia de descarga: debe contemplar la eficiencia de descarga de la batería, la eficiencia del inversor, la del regulador de carga en caso de que tenga salida de CC, pérdidas en cables, etc. Por defecto, puede usarse un valor del $75 \%$. 


$$
C_{n b}=\frac{E_{C T} *\left(D_{\text {out }}+1\right)}{V_{\text {nom }} * P_{D \max } * \eta_{D}} \quad(\text { Ecuación 11) }
$$

Donde $C_{n b}$ es la capacidad nominal del banco de baterías en Ah/día, $E_{C T}$ es el consumo energético diario total de la carga, $D_{\text {out }}$ son los días de autonomía con baja o nula insolación, $V_{\text {nom }}$ es la tensión nominal del sistema, $P_{D \max }$ es la profundidad máxima de descarga de la batería y $\eta_{D}$ es la eficiencia de la descarga.

\section{Cantidad de baterías:}

$$
N_{B T}=\frac{V_{\text {nom }} * C_{\text {nom banco }}}{V_{\text {nom_bat }} * C_{\text {nom_bat }}} \quad(\text { Ecuación 12) }
$$

Donde $N_{B T}$ es el número total de baterías del banco, $C_{\text {nom .banco es la capacidad nominal del banco, }}$ $C_{\text {nom_bat }}$ es la capacidad nominal de una batería, $V_{\text {nom }}$ es la tensión nominal del sistema y $V_{\text {nom_bat }}$ es la tensión nominal de una sola batería.

\section{Cantidad de baterías en serie:}

$$
N_{B S}=\frac{V_{\text {nom }}}{V_{\text {bat_nom }}}
$$

(Ecuación 13)

\section{Cantidad de baterías en paralelo:}

$$
N_{B P}=\frac{N_{B T}}{N_{B S}}
$$

(Ecuación 14)

\section{Dimensionamiento del regulador de carga:}

El regulador se conecta en serie con los paneles fotovoltaicos, por lo que circulará por él la corriente generada por ellos. Como regla de diseño, la corriente nominal del regulador se elige un $20 \%$ o $25 \%$ mayor a la corriente de cortocircuito ( $N_{p p} * I_{c c}$ ) entregada por el bloque generador o el mayor valor de la corriente de carga de continua ( $I_{\text {carga_cc }}$.

$I_{r e g}=1.25 * \max \left(N_{p p} * I_{c c} * I_{c a r_{-} c c}\right)($ Ecuación 15)

Donde $I_{\text {reg }}$ es la corriente nominal del regulador, $N_{p p}$ es el número de paneles solares en paralelo, $I_{c c}$ es la corriente de corto circuito de un panel fotovoltaico, $N_{p p} * I_{c c}$ es la corriente de corto circuito del bloque generador y para $\max \left(N_{p p} * I_{c c} * I_{c a r_{-} c c}\right)$ debe utilizarse el valor máximo entre la corriente de corto circuito del bloque generador y la demandada por la carga continua.

\section{Dimensionamiento del inversor:}

La potencia del inversor vendrá determinada en función de la potencia de los aparatos de consumo de CA, el rendimiento del mismo inversor y la simultaneidad de uso de dichos aparatos.

$$
\begin{array}{ll}
S_{\text {inv_out }}=1.25 * S_{\text {car } C A} * F S & (\text { Ecuación 16) } \\
S_{\text {inv_out }}=1.25 * \frac{P_{c a r C A}}{F p} * F S & (\text { Ecuación 17) } \\
P_{\text {inv_in }}=1.25 * \frac{P_{c a r C A}}{\eta_{\text {inv }}} * F S & (\text { Ecuación 18) } \\
P_{\text {inv_in }}=\frac{P_{\text {invout }}}{\eta_{\text {inv }}} * F p * F S & (\text { Ecuación 19) }
\end{array}
$$

Donde $S_{\text {inv_out }}$ es la potencia nominal del inversor, $P_{\text {inv_in }}$ es la potencia de entrada del inversor, $P_{\text {car }}$ es la potencia de las cargas en CA, Fp es el factor de potencia de las cargas en CA, FS es el factor de simultaneidad del consumo en CA y $\eta_{i n v}$ es el rendimiento del inversor.

Para tener en cuenta este hecho se aplica un factor de simultaneidad (FS), que representa la probabilidad de utilización simultánea de los aparatos de consumo de CA.

El valor de este coeficiente resulta de una estimación debida a la experiencia o a una reglamentación.

\section{Aplicación de caso}

El siguiente caso práctico de aplicación se realiza para una vivienda en Bogotá donde el consumo promedio de energía por persona está alrededor de los $1.6 \mathrm{KWh} /$ día; es decir, está por encima de los $6 \mathrm{KWh}$ /día para una familia de cuatro personas según los indicadores de consumo de energía eléctrica per cápita del Banco Mundial. 
En este caso el sistema fotovoltaico se va a dimensionar solo para algunos electrodomésticos de la vivienda, ya que la idea no es desconectar de la red la vivienda sino apoyar con el sistema fotovoltaico el consumo energético de esta.

Los electrodomésticos son los siguientes:

1. Cinco bombillos de bajo consumo de $10 \mathrm{~W}$.

2. Un computador portátil de $90 \mathrm{~W}$.

3. Dos celulares de $10 \mathrm{~W}$.

4. Un decodificador digital de $30 \mathrm{~W}$.

5. Un televisor de $40^{\prime \prime}$ de $100 \mathrm{~W}$.

No se prevé realizar una instalación de CC.

Se requiere una alta confiabilidad del sistema en lo que respecta a días con baja irradiancia (nublados).

\section{Estimación del consumo:}

Tabla 1. Estimación del consumo eléctrico de los electrodomésticos en Wh/día.

\begin{tabular}{|c|c|c|c|c|}
\hline Dispositivo & Cantidad & Potenda(W) & Horas(h) & $\begin{array}{c}\text { Consumo } \\
\text { total(Wh/dia) }\end{array}$ \\
\hline bombillos & 5 & 10 & 6 & 300 \\
\hline PC portátil & 1 & 90 & 3 & 270 \\
\hline $\begin{array}{c}\text { Decodificador } \\
\text { digital }\end{array}$ & 1 & 30 & 4 & 120 \\
\hline Celulares & 2 & 10 & 2 & 40 \\
\hline Televisor & 1 & 100 & 4 & 400 \\
\hline \multicolumn{6}{|c|}{ Consumo total promedio (Wh/dia) } \\
\hline
\end{tabular}

Fuente: Elaboración propia.

Como se puede apreciar en la tabla anterior, el consumo para calcular el sistema es de $1130 \mathrm{Wh} /$ día.

$$
E_{\text {Cargatotal }}=E_{A C}=1130 \mathrm{Wh} / \text { día. }
$$

\section{Estimación de pérdidas:}

- $\quad \eta B$ : eficiencia de las baterías (80\%).

- $\quad$ inv: eficiencia del inversor (85\%).

- $\quad \eta R$ : eficiencia del regulador (95\%).
- $\quad$ \X: eficiencia debida a otras pérdidas (95\%)

Cálculo de la eficiencia:

$$
\begin{gathered}
\eta_{T}=0.8 * 0.85 * 0.95 * 0.95 \\
\eta_{T} \approx 0.614
\end{gathered}
$$

El bloque generador deberá generar 39\% más energía para compensar las pérdidas.

\section{Calculo del ángulo óptimo de inclinación de los paneles:}

Mediante las coordenadas geográficas de Bogotá en la página de la $\mathrm{Nasa}^{2}$ se obtienen los datos de insolación promedio mínima para cada mes a distintas inclinaciones. Por su ubicación cercana al ecuador, el ángulo de inclinación puede estar entre $0^{\circ}$ y $20^{\circ}$ en orientación al sur.

Tabla 3. Descripción del panel.

\begin{tabular}{|l|c|c|}
\hline \multicolumn{3}{|c|}{150 W Polycrystaline Silicon Solar panel } \\
\hline Potencia nominal & $150 \mathrm{~W}$ & Wp \\
\hline Tencion a PN & 18,5 & V \\
\hline Corriente a PN & 8,5 & A \\
\hline Tencion circuito abierto & 22,9 & V \\
\hline Corriente de corto circuito & 8,757 & $\mathrm{~A}$ \\
\hline
\end{tabular}

Fuente: Elaboración propia.

\section{Energía a generar por el bloque generador:}

$$
\begin{gathered}
E_{\text {gen }}=1.1\left(\frac{E_{\text {CargaTotal }}}{\eta_{T}}\right) \\
E_{\text {gen }}=1.1\left(\frac{1130 \mathrm{Wh} / \text { dia }}{0,614}\right) \\
E_{\text {gen }} \approx 2024,43 \mathrm{Wh} / \text { dia }
\end{gathered}
$$

Energía diaria generada por un panel de 150 Wp sin MPPT.

2. Véase https://eosweb.larc.nasa.gov/sse/. 
Para el cálculo se necesita conocer algunos parámetros climáticos del lugar, las horas solares pico (HSP), la potencia pico del panel (Wp) y la tensión pico $(\mathrm{Vp})$.

Este cálculo se realiza dividiendo el valor de radiación solar de la peor época del año sobre 1 kw/ $\mathrm{m} 2$ y de esta forma se obtienen las horas solares pico para la producción de energía.

$$
\begin{aligned}
& \mathrm{HSP}=\left(4.5 \frac{\mathrm{kWh}}{\mathrm{m}^{2}} / \text { día }\right) /\left(1 \frac{\mathrm{kW}}{\mathrm{m}^{2}}\right) \approx 4.5 \mathrm{~h} / \text { día } \\
& \mathrm{Wp}=150 \mathrm{~W} \\
& \mathrm{Vp}=18.5 \mathrm{~V} \\
& E_{\text {panel }}=W_{p(T)} * H S P=150 \mathrm{~W} * 4.5 \mathrm{~h} / \text { día } \\
& E_{\text {panel }}=675 \mathrm{Wh} / \text { día }
\end{aligned}
$$

\section{Energía extra de recuperación:}

En este caso de considerará generar un 20\% de energía extra para prevenir el riesgo de generación en días nublados.

$$
\begin{gathered}
E_{\text {Extra }}=\gamma_{\text {Extra }} * E_{\text {gen }} \\
E_{\text {Extra }}=0.2 * 2024,43 \mathrm{Wh} / \text { día } \\
E_{\text {Extra }} \approx 404.9 \mathrm{Wh} / \text { día }
\end{gathered}
$$

\section{Cantidad total de paneles:}

$$
\begin{gathered}
N_{T P}=\frac{E_{\text {gen }}+E_{E x t r a}}{E_{\text {panel }}} \\
N_{T P}=\frac{(2024.43+404.9) W h / \text { día }}{675 \mathrm{Wh} / \text { día }} \\
N_{T P}=|4| \\
E_{\text {panel }=N_{T P} * E_{\text {panel }}=N_{T P} * W_{p(T)} * H S P} \\
E_{\text {panel }}=4 * 150 \mathrm{~W} * \frac{4.5 \mathrm{~h}}{\text { dia }}=2700 \mathrm{Wh} / \text { día }
\end{gathered}
$$

\section{Dimensionamiento del banco de baterías:}

Dout: días de autonomía: 3 días y 4 noches.

PDmax: profundidad máxima de descarga $80 \%$. $\eta \mathrm{D}$ : eficiencia de descarga de batería 90\%.

$$
\begin{aligned}
& C_{n b}=\frac{E_{C T} *\left(D_{\text {out }}+1\right)}{V_{\text {nom }} * P_{\text {Dmax }} * \eta_{\text {inv }} * \eta_{D}} \\
& C_{n b}=\frac{1130 \mathrm{Wh} / \mathrm{dia}(3+1)}{24 \mathrm{~V} * 0.8 * 0.85 * 0.9} \\
& C_{n b}=308 \mathrm{Ah}
\end{aligned}
$$

Para el banco se utilizarán baterías de libre mantenimiento de $12 \mathrm{~V}$ y 220 Ah nominal.

Tabla 4. Descripción de tiempo de vida de las baterías.

\begin{tabular}{|c||c|c|}
\hline $\begin{array}{c}\text { Temperatura media } \\
\text { de funcionamiento }\end{array}$ & AGM(años) & Gel(años) \\
\hline $20^{\circ} \mathrm{C}$ & $7-10$ & 12 \\
\hline $30^{\circ} \mathrm{C}$ & 4 & 6 \\
\hline $40^{\circ} \mathrm{C}$ & 2 & 3 \\
\hline
\end{tabular}

Fuente: Elaboración propia.

\section{Número total de baterías:}

$$
\begin{gathered}
N_{B T}=\frac{V_{\text {nom }} * C_{\text {nom_banco }_{\text {bat }}}}{V_{\text {nom_bat }} * C_{\text {nom_bat }_{-}}} \\
N_{B T}=\frac{24 \mathrm{~V} * 308 \mathrm{Ah}}{12 \mathrm{~V} * 220 \mathrm{Ah}}=4
\end{gathered}
$$

\section{Cantidad de baterías en serie:}

$$
\begin{aligned}
& N_{B S}=\frac{V_{\text {nom }}}{V_{\text {bat_nom }}} \\
& N_{B S}=\frac{24 \mathrm{~V}}{12 \mathrm{~V}}=2
\end{aligned}
$$




\section{Cantidad de baterías en paralelo:}

$$
\begin{gathered}
N_{B P}=\frac{N_{B T}}{N_{B S}} \\
N_{B P}=\frac{4}{2}=2
\end{gathered}
$$

\section{Máxima corriente de carga y descarga del banco:}

A continuación, se calculará la corriente máxima de carga $C_{5}$ y de descarga $C_{20}$ para el banco de baterías calculado.

$$
\begin{aligned}
& C_{\text {max }_{-} \text {car }}=N_{B P} * C_{5}=2 \frac{220 \mathrm{Ah}}{5 h} \\
& C_{\text {max } d e s}=88 \mathrm{~A} \\
& C_{\text {max }_{-} \text {des }}=N_{B P} * C_{20}=2 \frac{220 \mathrm{Ah}}{20 \mathrm{~h}} \\
& C_{\text {max }_{-} \text {des }}=22 \mathrm{~A}
\end{aligned}
$$

\section{Dimensionamiento del regulador de carga MPPT:}

La potencia del generador fotovoltaico puede exceder la máxima potencia de entrada del mismo. En este caso, el regulador limitará dicha potencia, proporcionando la corriente nominal máxima especificada por el fabricante, de esta forma el dispositivo no sufrirá ningún daño. Una de las características de los reguladores MPPT es que tienen su punto de máxima eficiencia al trabajar en potencias cercanas a las nominales. Como el costo de los reguladores se incrementa con la potencia nominal del mismo, dimensionar un regulador basado en la potencia pico del generador conlleva un gasto innecesario.

Para el dimensionamiento del regulador de carga es necesario tener en cuenta la potencia pico del generador fotovoltaico y la tensión nominal del banco de baterías:

- Tensión nominal del banco de baterías: $24 \mathrm{~V}$.

- Potencia pico del generador fotovoltaico: 600 Wp $(4 \times 150 \mathrm{Wp})$.

- Tensión del circuito abierto de los paneles fotovoltaicos: $22.9 \mathrm{~V}$.

Tabla 5. Selección del regulador MTTP.

\begin{tabular}{|l|c|c|}
\hline \multicolumn{3}{|c|}{ Electrical TS-MPPT-45 } \\
\hline Maximun Battery Current & \multicolumn{2}{|c|}{$45 \mathrm{~A}$} \\
\hline \multirow{3}{*}{ Nominal Maximun Solar imput } & $12 \mathrm{~V}$ & $600 \mathrm{~W}$ \\
\cline { 2 - 3 } & $24 \mathrm{~V}$ & $1200 \mathrm{~W}$ \\
\cline { 2 - 3 } & $48 \mathrm{~V}$ & $2400 \mathrm{~W}$ \\
\hline Max. Solar Open circuit & \multicolumn{2}{|c|}{$150 \mathrm{~V}$} \\
\hline
\end{tabular}

Fuente: Elaboración propia.

Se utilizará un regulador MPPT de $45 \mathrm{~A}$ nominal y $1200 \mathrm{Wp}$ de potencia nominal, funcionando con un banco de baterías de $24 \mathrm{~V}$ nominal.

$$
\begin{aligned}
& V_{\text {in }}=1,25 * N_{P S} * V_{O C} \\
& V_{\text {in }}=1,25 * 4 * 22.9 \\
& V_{\text {in }}=114.5 \mathrm{~V}
\end{aligned}
$$

Para este caso, la máxima tensión de entrada no excede la máxima tensión de entrada del regulador de carga especificada por el fabricante (150 V).

\section{Máxima corriente de carga y descarga:}

El consumo de potencia total de la carga estará dado por la suma de potencia (nominal) de todos los componentes eléctricos en régimen permanente $y$ tiene un valor de $290 \mathrm{~W}$.

$$
I_{\text {Des_bat }}=1.25 \frac{P_{\text {Car_CA }_{-}}}{V_{\text {nom }} * \eta_{\text {inver }}}
$$




$$
I_{\text {Des_bat }}=1.25 \frac{290 \mathrm{~W}}{24 \mathrm{~V} * 0.85} \approx 14.2<22 \mathrm{~A}
$$

La máxima corriente de carga podrá calcularse con base en la potencia nominal del regulador para un banco de baterías de $24 \mathrm{~V}$.

$$
\begin{gathered}
I_{\text {Carga }}=\frac{P_{\text {Reg }}}{V_{\text {min_bat }}} \\
I_{\text {Carga }}=\frac{1200 \mathrm{~W}}{21 \mathrm{~V}} \approx 51.14 \mathrm{~A}<88 \mathrm{~A}
\end{gathered}
$$

En este caso, ni la corriente de carga ni la de descarga superan los valores de C20 y C5 del banco de baterías respectivamente.

\section{Dimensionamiento del inversor:}

Se utilizará un $\mathrm{FP}=0.8$ y un $\mathrm{FS}=1$, esto debido a que podrían usarse todos al tiempo.

$$
\begin{aligned}
& S_{\text {inv_out }}=1.25 * S_{\text {car } C A} * F S=1.25 * \frac{P_{\text {car }}}{F p} * F S \\
& S_{\text {inv_out }}=1.25 * \frac{290 \mathrm{~W}}{0.8} * 1=453.2 \mathrm{VA} \\
& I_{\text {pico_carga }}=\frac{290 \mathrm{~W}}{220 \mathrm{~V}} \approx 1.4 \mathrm{~A}
\end{aligned}
$$

Tabla 6. Características del inversor.

\begin{tabular}{|l|c|}
\hline \multicolumn{2}{|c|}{ Inversor TGP 24-600 de 600VA } \\
\hline Maxima Corriente & $11 \mathrm{~A}$ \\
\hline Energia continua & $600 \mathrm{~W}$ \\
\hline Eficiencia MAX & $92 \%$ \\
\hline
\end{tabular}

Fuente: Elaboración propia.

\section{Esquema del sistema:}

- Cuatro paneles de 150 W.

- Un regulador de carga de 45 A nominal con MPPT.
- Inversor de 24V y $600 \mathrm{VA}$.

- Cuatro baterías de 220 Ah.

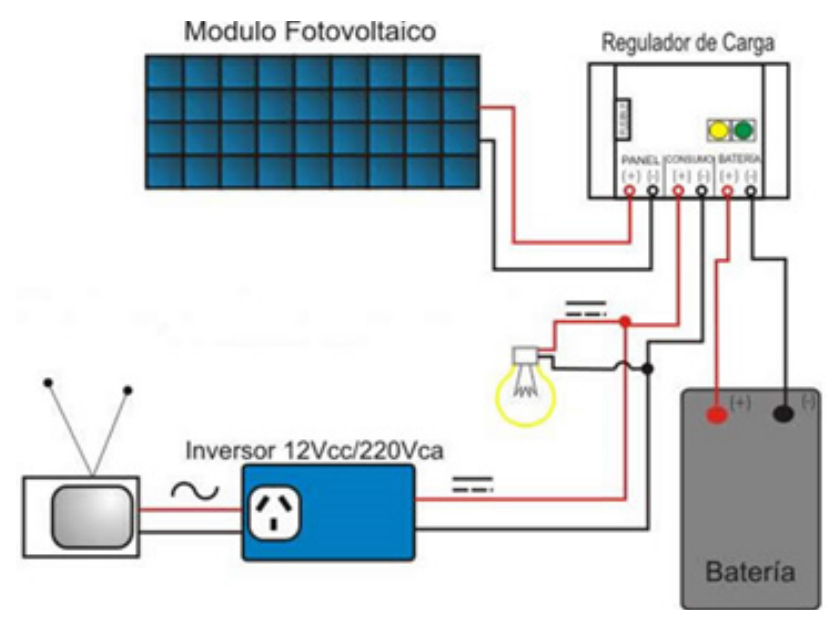

Figura 1. Esquema del sistema.

Fuente: Elaboración propia.

\section{Estimación de costos}

Los costos de instalación de un proyecto de energía fotovoltaica se ven representados por porcentajes en el siguiente esquema:

Tabla 8. Costo total de los principales componentes del sistema.

\begin{tabular}{|r|l|}
\hline Componente & Porcentaje \\
\hline Paneles solares & $22 \%-30 \%$ \\
\hline Baterías & $19 \%-35 \%$ \\
\hline Inversor & $10 \%-24 \%$ \\
\hline Regulador de carga & $6 \%-9 \%$ \\
\hline Materiales eléctricos & $2,5 \%-5 \%$ \\
\hline Montaje mecánico & $5 \%-9 \%$ \\
\hline Obra civil & $3 \%-15 \%$ \\
\hline Ingeniería y planificación & $3 \%-14 \%$ \\
\hline instalación & $10 \%-15 \%$ \\
\hline otros & $0 \%-2 \%$ \\
\hline
\end{tabular}

Fuente: Elaboración propia.

La tabla 7 presenta los porcentajes relativos de costo de una instalación fotovoltaica, brindada por los conocimientos del ingeniero Eugeni García, docente de ingeniería electrónica de la 
universidad Politécnica de Valencia en España. Se puede apreciar que la mayor parte de la inversión está en la adquisición de los paneles, el inversor, el regulador de carga MPPT y las baterías.

El coste real del proyecto varía con respecto a la selección de equipos, la aplicación y lugar en el cual se instale. Es por esta razón que los sistemas de montaje dependen del lugar de instalación del proyecto y de esto que el precio final de la instalación se acerque al costo de la adquisición de los principales sistemas eléctricos y electrónicos del proyecto; o, por otro lado, se trate de una instalación que requiera demasiada obra civil y el montaje de los paneles demande un sistema de anclaje especial para el lugar en el cual se van a instalar.

El porcentaje de los costos de instalación que corresponden a la obra civil y montaje mecánico hace referencia específicamente a lo que corresponde a adecuación del lugar y estructura, respectivamente, sobre la cual se hará el montaje del sistema fotovoltaico. Como el costo real de esta parte corresponde a un valor el cual podría ser exclusivo para cada proyecto, dadas las condiciones del lugar y en el cual se hará la instalación, no se presentan los valores de obra civil y montaje mecánico para realizar los cálculos de retorno de inversión.

Basado en cálculos de PRI (Periodo de retorno de inversión), en el cual se hacen las siguientes estimaciones: la instalación del sistema fotovoltaico es realizado en la ciudad de Bogotá donde el precio de $\mathrm{kW} / \mathrm{h}$ de energía es vendido a 450.50 $\mathrm{COP}$, decimos que el precio total del proyecto de energía fotovoltaica es de cinco millones de pesos. También realizamos el cálculo basándonos en la factura de energía de una vivienda donde el consumo promedio es de $169 \mathrm{kWh} / \mathrm{mes}$, además, según los cálculos de los paneles seleccionados el sistema es capaz de proveer $81 \mathrm{kWh} / \mathrm{mes}$, pero solo se van a aprovechar realmente $34 \mathrm{kWh} /$ mes los cuales son el consumo mensual de los elementos conectados al sistema, entonces podemos decir que vamos a dejar de pagar (34 kWh/mes) $x(450.50 \mathrm{COP})=15.317$ COP al mes con una instalación de este tipo.

También, conociendo que al término de 10 años hay que realizar un cambio de baterías, lo cual genera otra inversión en el periodo de funcionamiento del proyecto, se realizan los cálculos de la siguiente manera:

Tabla 8. Costo total de los principales componentes del sistema.

\begin{tabular}{|l|l|r|r|}
\hline componentes & Despcripción & Precio unidad & Precio Total \\
\hline Paneles Solares & 150 W Polycrystaline Silicon Solar panel & 375000 & 1500000 \\
\hline Baterias & Batería GEL Victron 12V 220 Ah & 520000 & 2080000 \\
\hline Inversor & Inversor TGP 24-600 de 600VA & 540000 & 540000 \\
\hline Regulador MPPT & Electrical TS-MPPT-45 & 300000 & 300000 \\
\hline \multicolumn{2}{|l|}{} & Total & 4420000 \\
\hline
\end{tabular}

Fuente: Elaboración propia.

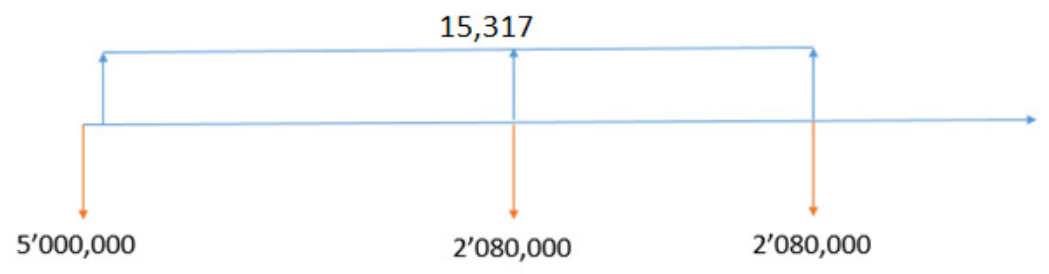

Figura 2. Flujo de caja del proyecto.

Fuente: Elaboración propia. 
De los cálculos de PRI obtenemos que para el periodo de instalación del sistema fotovoltaico, correspondiente a 25 años, en los cuales se hace respectivamente el cambio de baterías a los 10 y 20 años, los cuales generan otras dos inversiones correspondientes al valor de adquisición de las baterías, luego de los 25 años no se logra recuperar la inversión, por el contrario nos encontramos con que al final del periodo de instalación del sistema existe un déficit correspondiente por un poco menos de 3 millones de pesos los cuales corresponden al valor de inversión de los cambios de baterías en los dos periodos contemplados. Por otro lado, hay que contemplar los costos de mantenimiento que un sistema como este requiere, el cual se reduce a la limpieza de los paneles y red eléctrica del sistema. Así, el costo de mantenimiento contemplando estos dos factores no es muy elevado, sin embargo, se debe tener en cuenta ya que uno de los factores de eficiencia para el correcto funcionamiento de los paneles depende de que la superficie de los paneles esté limpia.

\section{Conclusiones}

Con los resultados obtenidos de los cálculos realizados para el dimensionamiento del sistema fotovoltaico, el cual está diseñado para una vivienda ubicada en Bogotá, es posible observar que sí es viable desde el punto de vista técnico implementar dicho sistema. Con los datos obtenidos de la radiación de la ciudad fue posible dimensionar un sistema el cual, aunque es un poco robusto debido a que el diseño está basado en el peor dato de radiación solar que llega a la ciudad (la cantidad de horas pico de sol y el tiempo de autonomía del sistema), se trata de un sistema confiable ya que proporciona la energía necesaria para tres días y cuatro noches de autonomía sin recibir radiación solar durante este periodo.

El dimensionamiento del sistema solar tiene gran cantidad de variables, las cuales están a disposición del diseñador, por lo cual la elección de estos componentes debe hacerse teniendo en cuenta las necesidades de diseño y, adicionalmente, hay que tener en cuenta el aspecto económico del proyecto. Ya que podemos tener infinitas configuraciones del sistema dependiendo, por ejemplo, de los paneles solares que escojamos, ya que el precio de los paneles se eleva considerablemente a medida que sea más grande el valor de potencia nominal.

Además de esto, se debe considerar el mantenimiento del sistema de producción de energía fotovoltaica. Donde tenemos que los paneles solares están construidos para una duración de 25 años y no necesitan mayor cuidado que la limpieza de polvo. Por otro lado, están las baterías, las cuales tienen un tiempo de vida promedio de 10 años, lo que quiere decir que, aunque los paneles duren 25 años, las baterías tendrán que ser reemplazadas por unas nuevas luego de 10 años de uso. Con el resto de sistemas eléctricos y electrónicos es pertinente saber que van a tener algún tipo de desgaste y que por esta degradación de los componentes se pierde la eficiencia que se tiene al principio de la instalación.

Por último, de la estimación de costos se concluye que un proyecto de este tipo, donde transcurren los 25 años previstos de funcionamiento, en ningún momento cabe la posibilidad de recuperar la inversión inicial, mucho menos de generar un ingreso proporcionado por la implementación de este sistema. Tenemos que no es viable el proyecto desde el punto de vista económico, ya que lo que va a generar realmente es un gasto adicional. Pero si lo que se está buscando realmente es contribuir con el cambio climático, como se pudo apreciar, es posible implementar algún tipo de sistema fotovoltaico en la ciudad de Bogotá y de esta forma suplir a la población con una parte de la energía eléctrica que se consume.

\section{Referencias}

Abella, M. A. (2016). 13. Dimensionado de Sistemas Fotvoltaicos: Otros métodos de dimensionado de sistemas FV autónomos. Centro 
de investigaciones Energeticas, Medioambientales y Tecnológicas, Departamento de Energia Renovables, Ciemat. Recuperado de: http://api.eoi.es/api_v1 dev.php/fedora/asset/ eoi:45340/componente45338.pdf

Aguilar, F. J., Aledo, S. y Quiles, P. V. (2016). Experimental study of the solar photovoltaic contribution for the domestic hot water production with heat pumps in dwellings. Applied Thermal Engineering, 101, 1-11. Doi: 10.1016/j. applthermaleng.2016.01.127.

Al-Shohani, W. A. M., Al-Dadah, R. y Mahmoud, S. (2016). Reducing the thermal load of a photovoltaic module through an optical water filter. Applied Thermal Engineering, 109, 475-486. Doi: 10.1016/j. applthermaleng.2016.08.107.

Ben, M. y Ben, S. (2017). The role of renewable energy and agriculture in reducing $\mathrm{CO} 2$ emissions : Evidence for North Africa countries. Ecological Indicators, 74, 295-301. Doi: 10.1016/j.ecolind.2016.11.032.

Cao, Y., Liu, C., Huang, Y., Wang, T., Sun, C. y Yuan, Y. (2017). Parallel algorithms for islanded microgrid with photovoltaic and energy storage systems planning optimization problem : Material selection and quantity demand optimization. Computer Physics Communications, 211, 45-53. Doi: 10.1016/j.cpc.2016.07.009.

Chandel, S. S., Nagaraju Naik, M. y Chandel, R. (2015). Review of solar photovoltaic water pumping system technology for irrigation and community drinking water supplies. Renewable and Sustainable Energy Reviews, 49, 10841099. Doi: 10.1016/j.rser.2015.04.083.

Fouda, A., Nada, S. A. y Elattar, H. F. (2016). An integrated $\mathrm{A} / \mathrm{C}$ and $\mathrm{HDH}$ water desalination system assisted by solar energy: Transient analysis and economical study. Applied Thermal Engineering, 108, 1320-1335. Doi: 10.1016/j. applthermaleng.2016.08.026.

García, M. E. (2016). Energia solar fotovoltaica aislada. Applied Solar Energy, curso de energias renovables. Universidad Politecnica de
Valencia. Recuperado de: www.cursofotovoltaica.com

Gasparatos, A., Doll, C. N. H., Esteban, M., Ahmed, A. y Olang, T. A. (2017). Crossmark. Renewable and Sustainable Energy Reviews, 70, 161-184. Doi: 10.1016/j.rser.2016.08.030.

Ghasemi Mobtaker, H., Ajabshirchi, Y., Ranjbar, S. F. y Matloobi, M. (2016). Solar energy conservation in greenhouse: Thermal analysis and experimental validation. Renewable Energy, 96, 509-519. Doi: 10.1016/j. renene.2016.04.079.

Islam, M. M., Pandey, A. K., Hasanuzzaman, M. y Rahim, N. A. (2016). Recent progresses and achievements in photovoltaic-phase change material technology: A review with special treatment on photovoltaic thermal-phase change material systems. Energy Conversion and Management, 126, 177-204. Doi: 10.1016/j. enconman.2016.07.075.

Kannan, N. y Vakeesan, D. (2016). Solar energy for future world: A review. Renewable and Sustainable Energy Reviews, 62, 1092-1105. Doi: 10.1016/j.rser.2016.05.022.

Lewis, N. S. (2007). Toward Cost-Effective Solar Energy Use. Science, 315(5813), 798-801. Doi: 10.1126/science.1137014

Lúcio, G., Filho, T., Adriano, C., Mambeli, R., Felipe, I., Dos, S. y Braga, G. (2016). Solar Energy Materials \& Solar Cells Study of the energy balance and environmental liabilities associated with the manufacture of crystalline Si photovoltaic modules and deployment in different regions. Solar Energy Materials and Solar Cells, 144, 383-394. Doi: 10.1016/j. solmat.2015.09.023.

Muhsen, D. H., Ghazali, A. B. y Khatib, T. (2016). Multiobjective differential evolution algorithm-based sizing of a standalone photovoltaic water pumping system. Energy Conversion and Management, 118, 32-43.Doi: 10.1016/j. enconman.2016.03.074.

Muhsen, D. H., Ghazali, A. B., Khatib, T., Abed, I. A. y Natsheh, E. M. (2016). Sizing of a 
standalone photovoltaic water pumping system using a multi-objective evolutionary algorithm. Energy, 109, 961-973. Doi: 10.1016/j. energy.2016.05.070.

Olaya, Y., Arango-Aramburo, S. y Larsen, E. R. (2016). How capacity mechanisms drive technology choice in power generation: The case of Colombia. Renewable and Sustainable Energy Reviews, 56, 563-571. Doi: 10.1016/j. rser.2015.11.065.

Peralta, R. (2011). La energía solar fotovoltaica como factor de desarrollo en zonas rurales de Colombia. caso: vereda Carupana, municipio de Tauramena, departamento de Casanare. Tesis de Maestría en Desarrollo Rural, Facultad de Estudios Ambientales y Rurales, Pontificia Universidad Javeriana.

Rosso-Cerón, A. M. y Kafarov, V. (2015). Barriers to social acceptance of renewable energy systems in Colombia. Current Opinion in Chemical Engineering, 10, 103-110. Doi: 10.1016/j. coche.2015.08.003.

Senturk, A. y Eke, R. (2017). A new method to simulate photovoltaic performance of crystalline silicon photovoltaic modules based on datasheet values. Renewable Energy, 103, 5869. Doi: 10.1016/j.renene.2016.11.025.

Valer, L. R., Melendez, T. A., Fedrizzi, M. C., Zilles, R. y de Moraes, A. M. (2016). Variable-speed drives in photovoltaic pumping systems for irrigation in Brazil. Sustainable Energy Technologies and Assessments, 15, 20-26. Doi: 10.1016/j.seta.2016.03.003.

Wahyuni, N. S., Wulandari, S., Wulandari, E. y Pamuji, D. S. (2015). Integrated Communities for the Sustainability of Renewable Energy Application: Solar Water Pumping System in Banyumeneng Village, Indonesia. Energy Procedia, 79, 1027-1032. Doi: 10.1016/j. egypro.2015.11.604.

Yahyaoui, I., Chaabene, M. y Tadeo, F. (2015). Evaluation of Maximum Power Point Tracking algorithm for off-grid photovoltaic pumping. Sustainable Cities and Society, 25, 65-73. Doi: 10.1016/j.scs.2015.11.005.

Zhou, J., Zhang, Z., Liu, H. y Yi, Q. (2017). Temperature distribution and back sheet role of polycrystalline silicon photovoltaic modules. Applied Thermal Engineering, 111, 1296-1303. Doi: 10.1016/j.applthermaleng.2016.10.095. 